\title{
State Estimation And Control FOR Systems with Perspective Outputs:
}

\section{TECHNICAL REPORT*}

\author{
João P. Hespanha \\ hespanha@ece.ucsb.edu \\ Dept. Electrical \&3 Computer Engineering, Univ. of California \\ Santa Barbara, CA 93106-9560
}

February 27, 2002

\begin{abstract}
In this paper we consider the problem of estimating the state of a system with perspective outputs. We formulate the problem in a deterministic setting by searching for the value of the state that is "most compatible" with the dynamics, in the sense that it requires the least amount of noise to explain the measured output. We show that, under appropriate observability assumptions, the optimal estimate converges globally to the true value of the state and can be used to design output-feedback controllers by using the estimated state to drive a state-feedback controller. We apply these results to the estimation of position and orientation of a controlled rigid body, using measurements from a charged-coupled-device camera attached to the body.
\end{abstract}

\section{Introduction}

This paper deals with dynamical systems with perspective outputs, inspired by the perspective systems introduced in [5]. Ghosh et al. [5] considered systems with linear dynamics and a homogeneous output function, whereas here we consider systems that are affine on the state but possibly nonlinear on the input. We also consider multiple homogeneous output functions. Formally, the systems under consideration are of the form

$$
\begin{aligned}
\dot{x} & =A(u) x+b(u)+G(u) \mathbf{d}, \\
\alpha_{j} y_{j} & =C_{j}(u) x+d_{j}(u)+\mathbf{n}_{j},
\end{aligned} \quad j \in\{1,2, \ldots, k\},
$$

where $x \in \mathbb{R}^{n}$ denotes the state of the system, $u \in \mathbb{R}^{n_{u}}$ its control input, $y_{j} \in \mathbb{R}^{m_{j}}$ its $j$ th perspective output, $\mathbf{d} \in \mathbb{R}^{n_{d}}$ an input disturbance that cannot be measured, and $\mathbf{n}_{j} \in \mathbb{R}^{n_{n}}$ measurement noise affecting the $j$ th output. Each $\alpha_{j} \in \mathbb{R}, j \in\{1,2, \ldots, k\}$ denotes a scalar that is determined by a normalization constrain such as

$$
\left\|y_{j}\right\|=1 \quad \text { or } \quad v_{j}^{\prime} y_{j}=1,
$$

where $v_{j} \in \mathbb{R}^{m_{j}}$ denotes a constant vector. When the matrices $A, b$, and all the $C_{j}, d_{j}$ are constant, and $\mathbf{d}$ and all the $\mathbf{n}_{j}$ are zero, we essentially have a perspective linear system in the sense of [5]. We call (1)-(2) a state-affine system with multiple perspective outputs, or for short simply a system with perspective outputs.

* Short version to appear in the 41th IEEE Conf. on Dec. and Control, 2002. This material is based upon work supported by the National Science Foundation under Grant No. ECS-0093762. 
Systems with perspective outputs typically arise when charged-coupled-device (CCD) cameras are used to acquire information about the position and orientation of moving rigid bodies. In Section 5 we will consider the specific problem of estimating the position and orientation of a controlled rigid body using measurements from a CCD camera attached to it. The dynamics of this system can be written as (1)-(2). The reader is referred to $[5,6,18]$ for several other examples of perspective systems in the context of motion and shape estimation.

This paper addresses state-estimation for systems with perspective outputs. In the last few years, the observability of perspective linear systems has been systematically studied in the literature and [4] provides an elegant algebraic observability test. It should be noted that for perspective linear systems without inputs it is never possible to recover the norm of the state because the system is homogeneous on the initial conditions. Therefore Dayawansa et al. [4] only consider state indistinguishability up a homogeneous scaling of the state. However, as shown in [7], for perspective systems with inputs it is in principle possible to recover the whole state from projective outputs. This is pursued here.

The main contribution of this paper is the design of a state-estimator for (1)-(2) (cf. Section 2). We propose an optimization approach towards state-estimation by defining the optimal state estimate $\hat{x}$ at time $t$ to be the value for the state of (1) that is compatible with the observations (2) collected up to time $t$ and the dynamics (1) for the "smallest" possible noise $\mathbf{n}_{j}$ and disturbances $\mathbf{d}$. This formulation is purely deterministic but leads to a state-estimator that resembles a Kalman-Bucy filter. In fact, if the same approach was applied to a linear system with linear outputs, one would arrive precisely at the Kalman-Bucy filter that would be obtained in a stochastic setting. The estimator proposed has the desirable property that, under suitable observability assumptions, the state-estimate converges asymptotically to its true value in the absence of noise and is bounded away from it when there is noise (cf. Section 3). We can therefore use this state-estimator to design output-feedback controllers by using the estimated state to drive state-feedback controllers (cf. Section 4).

A fundamental problem in mobile robotics is the determination of the position and orientation of a robot with respect to an inertial coordinate system. A promising solution to this problem is to utilize a camera mounted on the robot that observes the apparent motion on the image of stationary points. The linear and angular velocities of the camera can be assumed known in its own coordinate system (possibly with errors due to noise) but not in the inertial coordinate system. This is quite reasonable in mobile robotics where the motion of the camera is determined by the applied control signals. The problem of estimating the position and orientation of a camera mounted on a rigid body from the apparent motion of point features has a long tradition in the computer vision literature. We are interested here in filtering-like or iterative algorithms that continuously improve the estimates as more data (i.e., images) are acquired and that are robust with respect to measurement noise. Following the lead of other research (cf., e.g., [11, 8, 15, 9, 14, 3] and references therein) we formulate this as a state-estimation problem and utilize the estimator derived in Section 2 to solve it. One of the main contributions of this paper is that-opposite what happens with most previous algorithms - the one proposed here is globally convergent provided that suitable observability assumptions are satisfied. These assumptions are independent of the initialization of the estimator and depend solely on the motion of the camera. Global convergence to the correct position and orientation is achieved in the absence of noise. When there is noise, the magnitude of the estimation error is essentially proportional to the amount of noise (cf. Theorem 2). Another difference with respect to several other algorithms is that we also estimate scale. This can be achieved either through known (scaling) information about the points observed and/or through the knowledge of the camera's linear velocity. We also consider singular configurations for the points under observation, e.g., all points coplanar.

\section{State estimation}

For appropriate noise and disturbance signals, essentially every value for the state $x$ at a time $t \in \mathbb{R}$ is compatible with any outputs $y_{j}$ observed on the interval $[0, t)$. However, we will favor estimates for the state that can be made compatible with the measured outputs utilizing "small" noise and disturbance signals. In fact, we formulate state estimation as a deterministic optimization problem in which the estimate $\hat{x}(t)$ of the 
state at time $t \geq 0$ is the value for which the measured outputs can be made compatible with the system dynamics (1)-(2) for the "smallest" possible noise $\mathbf{n}_{j}$ and disturbance $\mathbf{d}$. Formally, given an input $u$ and measured outputs $y_{j}$ defined on an interval $[0, t)$, we define

$$
\hat{x}(t):=\arg \min _{z \in \mathbb{R}^{n}} J(z, t),
$$

where

$$
\begin{aligned}
J(z ; t):=\min _{\mathbf{d}, \mathbf{n}_{j}, \alpha_{j}}\left\{\int_{0}^{t}\left(\|\mathbf{d}\|^{2}+\sum_{j}\left\|\mathbf{n}_{j}\right\|^{2}\right) d \tau:\right. & \\
x(t)=z, \dot{x}=A(u) x+b(u)+G(u) \mathbf{d}, & \left.\alpha_{j} y_{j}=C_{j}(u) x+d_{j}(u)+\mathbf{n}_{j}\right\} .
\end{aligned}
$$

To avoid degeneracy, we add to $J$ a term penalizing the size of the state-estimate at time 0 . This corresponds to redefining $J$ as

$$
\begin{aligned}
J(z ; t):=\min _{\mathbf{d}, \mathbf{n}_{j}, \alpha_{j}}\left\{x(0)^{\prime} P_{0} x(0)\right. & \\
& +\int_{0}^{t}\left(\|\mathbf{d}\|^{2}+\sum_{j}\left\|\mathbf{n}_{j}\right\|^{2}\right) d \tau: \\
x(t)=z, \dot{x}=A(u) x+b(u)+G(u) \mathbf{d}, & \left.\alpha_{j} y_{j}=C_{j}(u) x+d_{j}(u)+\mathbf{n}_{j}\right\},
\end{aligned}
$$

with $P_{0}>0$. In practice, the term $x(0)^{\prime} P_{0} x(0)$ makes sure that any unobservable component of the state will be set to zero in the state estimate.

Remark 1. The approach just described towards state estimation can be viewed as the computation of a "generalized pseudo-inverse" that attempts to recover the current value of the state $x(t)$ from the measured output $y(\tau), \tau \in[0, t]$. To understand what is precisely meant by this let $U_{t}$ denote the triple consisting of the quantities that cannot be measured and that affect the value of the state at time $t$. Namely, the initial state, past noise and past disturbances:

$$
U_{t}:=\{x(0) ; \mathbf{n}(\tau), \tau \in[0, t] ; \mathbf{d}(\tau), \tau \in[0, t]\} .
$$

We denote by $\mathcal{U}_{t}$ the space of such triples. The system dynamics (1)-(2) define the following two operators

$$
\begin{aligned}
X_{t}: \mathcal{U}_{t} & \rightarrow \mathbb{R}^{n} & O_{t}: \mathcal{U}_{t} & \rightarrow \mathcal{Y}_{t} \\
U_{t} & \mapsto x(t) & U_{t} & \mapsto Y_{t}:=\left\{y_{j}(\tau), \tau \in[0, t], j=1,2, \ldots, k\right\}
\end{aligned}
$$

where $\mathcal{Y}_{t}$ denotes the appropriate output space. One can then view state estimation as solving the following system of equations

$$
\hat{x}=X_{t}\left(\hat{U}_{t}\right), \quad Y_{t}=O_{t}\left(\hat{U}_{t}\right),
$$

for the unknowns $\hat{x}$ and $\hat{U}_{t}$. If the observation operator $O_{t}$ had a left inverse $O_{t}^{-1}$ (e.g., in the absence of noise and if the system was observable) the solution to (6) would be unique and given by

$$
\hat{x}=X_{t}\left(O_{t}^{-1}\left(Y_{t}\right)\right) .
$$

However, in general this is not the case and the approach we propose is to replace the left inverse of $O_{t}$ by its "pseudo-inverse." In particular, we define the estimate $\hat{x}$ to be

$$
\hat{x}=X_{t}\left(O_{t}^{\perp}\left(Y_{t}\right)\right),
$$


where $O_{t}^{\perp}\left(Y_{t}\right)$ denotes the min-norm solution to $Y_{t}=O_{t}\left(U_{t}\right)$, i.e.,

$$
O_{t}^{\perp}\left(Y_{t}\right)=\arg \min _{\substack{U_{t} \in \mathcal{U}_{t}: \\ Y_{t}=O_{t}\left(U_{t}\right)}}\left\|U_{t}\right\|_{\mathcal{U}_{t}}
$$

The norm $\|\cdot\|_{\mathcal{U}_{t}}$ (or more precisely its square) is specified in the cost (5).

The following result solves the state-estimation problem defined above.

Theorem 1. The solution to the state-estimation problem defined by (4)-(5) is given by

$$
\begin{aligned}
\dot{Q} & =A(u) Q+Q A(u)^{\prime}+G(u) G(u)^{\prime}-Q W Q \\
\dot{\hat{x}} & =(A(u)-Q W) \hat{x}+b(u)-Q w
\end{aligned}
$$

with $Q(0)=P_{0}^{-1}, \hat{x}(0)=0$, where

$$
\begin{aligned}
W(t) & :=\sum_{j} C_{j}^{\prime}(u)\left(I-\frac{y_{j} y_{j}^{\prime}}{\left\|y_{j}\right\|^{2}}\right) C_{j}(u), & \\
w(t) & :=\sum_{j} C_{j}^{\prime}(u)\left(I-\frac{y_{j} y_{j}^{\prime}}{\left\|y_{j}\right\|^{2}}\right) d_{j}(u), & \forall t \geq 0 .
\end{aligned}
$$

Before proving Theorem 1 note that we can rewrite the state-estimation equation (8) as

$$
\begin{aligned}
& \dot{\hat{x}}=A(u) \hat{x}+b(u) \\
&+Q \sum_{j} C_{j}(u)^{\prime}\left(\hat{\alpha}_{j} y_{j}-C_{j}(u) \hat{x}-d_{j}(u)\right), \\
& \hat{\alpha}_{j}=\frac{y_{j}^{\prime}\left(C_{j}(u) \hat{x}+d_{j}(u)\right)}{\left\|y_{j}\right\|^{2}},
\end{aligned}
$$

which emphasizes the parallel between (8) and a Kalman-Bucy filter for linear systems.

Proof of Theorem 1. The function $J(z, t), z \in \mathbb{R}^{n}, t \geq 0$ can be viewed as a cost-to-go and computed using dynamics programing. To derive the dynamic programing operator we can consider an elementary time interval $d t$ and write ${ }^{1}$

$$
\begin{gathered}
J(z ; t)=\min _{\mathbf{d}, \alpha_{j}}\left\{x(0)^{\prime} P_{0} x(0)+\left(\|\mathbf{d}\|^{2}+\sum_{j}\left\|\alpha_{j} y_{j}-C_{j} z-d_{j}\right\|^{2}\right) d t\right. \\
+\int_{0}^{t-d t}\left(\|\mathbf{d}\|^{2}+\sum_{j}\left\|\alpha_{j} y_{j}-C_{j} x-d_{j}\right\|^{2}\right) d \tau: \\
x(t-d t)=z-(A z+b+G \mathbf{d}) d t, \dot{x}=A x+b+G \mathbf{d}\} \\
=\min _{\mathbf{d}, \alpha_{j}}\left(\|\mathbf{d}\|^{2}+\sum_{j}\left\|\alpha_{j} y_{j}-C_{j} z-d_{j}\right\|^{2}\right) d t+J(z-(A z+b+G \mathbf{d}) d t ; t-d t) .
\end{gathered}
$$

Subtracting $J(z ; t-d t)$ from both sides of the above equation, dividing by $d t$, and taking the limit as $d t \rightarrow 0$,

\footnotetext{
${ }^{1}$ For simplicity of notation, we will drop the dependence on $u$ of the matrices $A, b, G, C_{j}$ and $d_{j}$.
} 
leads to

$$
\begin{aligned}
& J_{t}(z ; t)= \min _{\mathbf{d}, \alpha_{j}}\|\mathbf{d}\|^{2}+\sum_{j}\left\|\alpha_{j} y_{j}-C_{j} z-d_{j}\right\|^{2}-J_{z}(z ; t)(A z+b+G \mathbf{d}) \\
&=\min _{\mathbf{d}, \alpha_{j}} \| \mathbf{d}- \frac{1}{2} G^{\prime} J_{z}(z ; t)^{\prime}\left\|^{2}-\frac{1}{4}\right\| G^{\prime} J_{z}(z ; t)^{\prime} \|^{2}-J_{z}(z ; t)(A z+b) \\
& \quad+\sum_{j}\left\|y_{j}\right\|^{2} \alpha_{j}^{2}-2 y_{j}^{\prime}\left(C_{j} z+d_{j}\right) \alpha_{j}+\left\|C_{j} z+d_{j}\right\|^{2} \\
&=-\frac{1}{4}\left\|G^{\prime} J_{z}(z ; t)^{\prime}\right\|^{2}-J_{z}(z ; t)(A z+b) \quad+\min _{\alpha_{j}} \sum_{j}\left\|y_{j}\right\|^{2}\left(\alpha_{j}-\frac{y_{j}^{\prime}\left(C_{j} z+d_{j}\right)}{\left\|y_{j}\right\|^{2}}\right)^{2}+\left(C_{j} z+d_{j}\right)^{\prime}\left(I-\frac{y_{j} y_{j}^{\prime}}{\left\|y_{j}\right\|^{2}}\right)\left(C_{j} z+d_{j}\right) \\
& \\
&=-\frac{1}{4}\left\|G^{\prime} J_{z}(z ; t)^{\prime}\right\|^{2}-J_{z}(z ; t)(A z+b)+\sum_{j}\left(C_{j} z+d_{j}\right)^{\prime}\left(I-\frac{y_{j} y_{j}^{\prime}}{\left\|y_{j}\right\|^{2}}\right)\left(C_{j} z+d_{j}\right),
\end{aligned}
$$

where $J_{t}$ and $J_{z}$ denote the partial derivatives of $J$ with respect to $t$ and $z$, respectively. The value of $J(z ; t)$ can then be determined from the linear partial differential equation (9) with initial condition

$$
J(z ; 0)=z^{\prime} P_{0} z, \quad z \in \mathbb{R}^{n} .
$$

It turns out that the solution to (9)-(10) can be written as

$$
J(z ; t)=(z-\hat{x}(t))^{\prime} P(t)(z-\hat{x}(t))+J_{0}(t),
$$

for appropriately defined signals $\hat{x}(t)$ and $J_{0}(t)$. The signal $\hat{x}$ is then precisely the estimate for the state $x$ of the perspective linear system. Moreover, matching (10) with (11) we conclude that

$$
\begin{array}{lll}
P(0)=P_{0}, & \hat{x}(0)=0, & J_{0}(0)=0 .
\end{array}
$$

To verify that the solution to (9)-(10) can indeed be written as (11), we replace this equation in (9) and obtain

$$
\begin{aligned}
& -2(z-\hat{x})^{\prime} P \dot{\hat{x}}+(z-\hat{x})^{\prime} \dot{P}(z-\hat{x})+\dot{J}_{0} \\
& \quad=-\left\|G^{\prime} P(z-\hat{x})\right\|^{2}-2(z-\hat{x})^{\prime} P(A z+b)+\sum_{j}\left(C_{j} z+d_{j}\right)^{\prime}\left(I-\frac{y_{j} y_{j}^{\prime}}{\left\|y_{j}\right\|^{2}}\right)\left(C_{j} z+d_{j}\right),
\end{aligned}
$$

or equivalently

$$
\begin{aligned}
z\left(\dot{P}+P A+A^{\prime} P+P G G^{\prime} P-\right. & W) z+2 z^{\prime}\left(-P \dot{\hat{x}}-\dot{P} \hat{x}-P G G^{\prime} P \hat{x}-A^{\prime} P \hat{x}+P b-w\right) \\
& +\dot{J}_{0}+2 \hat{x}^{\prime} P \dot{\hat{x}}+\hat{x}^{\prime} \dot{P} \hat{x}+\hat{x}^{\prime} P G G^{\prime} P \hat{x}-2 \hat{x}^{\prime} P b-\sum_{j} d_{j}^{\prime}\left(I-\frac{y_{j} y_{j}^{\prime}}{\left\|y_{j}\right\|^{2}}\right) d_{j}=0 .
\end{aligned}
$$

This equation holds provided that

$$
\begin{aligned}
& \dot{P}+P A+A^{\prime} P+P G G^{\prime} P-W=0 \\
& -P \dot{\hat{x}}-\dot{P} \hat{x}-P G G^{\prime} P \hat{x}+P b-A^{\prime} P \hat{x}-w=0 \\
& \dot{J}_{0}+2 \hat{x}^{\prime} P \dot{\hat{x}}+\hat{x}^{\prime} \dot{P} \hat{x}+\hat{x}^{\prime} P G G^{\prime} P \hat{x}-2 \hat{x}^{\prime} P b-\sum_{j} d_{j}^{\prime}\left(I-\frac{y_{j} y_{j}^{\prime}}{\left\|y_{j}\right\|^{2}}\right) d_{j}=0 .
\end{aligned}
$$


Replacing (12) in (13) and these two equations in (14), we obtain another set of equivalent equations:

$$
\begin{aligned}
-\dot{P} & =P A+A^{\prime} P+P G G^{\prime} P-W \\
P \dot{\hat{x}} & =P A \hat{x}+P b-W \hat{x}-w \\
\dot{J}_{0} & =\hat{x}^{\prime} W \hat{x}+\sum_{j} d_{j}^{\prime}\left(I-\frac{y_{j} y_{j}^{\prime}}{\left\|y_{j}\right\|^{2}}\right) d_{j} .
\end{aligned}
$$

It turns out that (15) can also be written as

$$
\dot{P}=-P\left(A+G G^{\prime} P\right)-\left(A+G G^{\prime} P\right)^{\prime} P+P G G^{\prime} P+W
$$

and therefore ${ }^{2}$

$$
P=\Psi(0, t)^{\prime} P_{0} \Psi(0, t)+\int_{0}^{t} \Psi(\tau, t)^{\prime}\left(P G G^{\prime} P+W\right) \Psi(\tau, t) d \tau, \quad t \geq 0
$$

where $\Psi(t, \tau)$ denotes the state transition matrix of $\dot{z}=\left(A+G G^{\prime} P\right) z$. Since $\Psi(t, 0) P_{0} \Psi(t, 0)^{\prime}>0$ and $P G G^{\prime} P+W \geq 0$, we conclude that $P(t)$ remains positive definite for all time. Therefore (16) is actually equivalent to (8) with $Q=P^{-1}$. Using the fact that $\dot{Q}=-Q \dot{P} Q$, it is straightforward to conclude that the matrix $Q$ can be generated directly from (7).

\section{Estimator convergence}

We are now interested in investigating under what conditions the state estimate $\hat{x}$ provided by Theorem 1 converges to the true state $x$ of the perspective system. The following technical assumption is needed:

Assumption 1. There exist positive constants $\delta, \Delta \in(0, \infty)$ such that $\delta I \leq G(u) G^{\prime}(u) \leq \Delta I, \forall u \in \mathbb{R}^{n_{u}}$.

This mild assumption essentially guarantees that $G(u)$ is bounded and full-row rank, "uniformly" over all possible inputs. The following result establishes the convergence of the state estimate.

Theorem 2. Assuming that the solution to the process (1)-(2) exists globally, the solution to state estimator (7)-(8) also exists globally. Moreover, when Assumption 1 holds and $Q$ remains uniformly bounded, there exist positive constants $c, \lambda, \gamma_{d}, \gamma_{1}, \ldots, \gamma_{k}$ such that

$$
\begin{array}{ll}
\|\tilde{x}(t)\| \leq c e^{-\lambda t}\|\tilde{x}(0)\|+\gamma_{d} \sup _{\tau \in(0, t)}\|\mathbf{d}(\tau)\| \\
\quad+\sum_{j} \gamma_{j} \sup _{\tau \in(0, t)}\left\|\mathbf{n}_{j}(\tau)\right\|, \quad \forall t>0,
\end{array}
$$

where $\tilde{x}:=\hat{x}-x$ denotes the state estimation error.

\footnotetext{
${ }^{2}$ This can be verified by taking derivatives of the candidate expression for $P$ :

$$
\begin{aligned}
\dot{P}= & -\left(A+G G^{\prime} P\right)^{\prime} \Psi(0, t)^{\prime} P_{0} \Psi(0, t)-\Psi(0, t)^{\prime} P_{0} \Psi(\tau, t)\left(A+G G^{\prime} P\right)+P G G^{\prime} P+W \\
& -\left(A+G G^{\prime} P\right)^{\prime} \int_{0}^{t} \Psi(\tau, t)^{\prime}\left(P G G^{\prime} P+W\right) \Psi(\tau, t) d \tau-\int_{0}^{t} \Psi(\tau, t)^{\prime}\left(P G G^{\prime} P+W\right) \Psi(\tau, t) d \tau\left(A+G G^{\prime} P\right) \\
=- & \left(A+G G^{\prime} P\right)^{\prime} P-P\left(A+G G^{\prime} P\right)+P G G^{\prime} P+W .
\end{aligned}
$$
}

Here we used the fact that, for every fixed $\tau$,

$$
\frac{\mathrm{d}}{\mathrm{d} t} \Psi(\tau, t)=-\Psi(t, \tau)^{-1}\left(\frac{\mathrm{d}}{\mathrm{d} t} \Psi(t, \tau)\right) \Psi(t, \tau)^{-1}=-\Psi(t, \tau)^{-1}\left(A+G G^{\prime} P\right) \Psi(t, \tau) \Psi(t, \tau)^{-1}=-\Psi(\tau, t)\left(A+G G^{\prime} P\right) .
$$


Proof of Theorem 2. From (1) and (8) we conclude that ${ }^{3}$

$$
\begin{aligned}
\dot{\tilde{x}} & =(A-Q W) \tilde{x}-G \mathbf{d}-Q \sum_{j} C_{j}^{\prime} Y_{j}^{\prime} Y_{j}\left(C_{j} x+d_{j}\right) \\
& =(A-Q W) \tilde{x}-G \mathbf{d}-Q \sum_{j} C_{j}^{\prime} Y_{j}^{\prime} Y_{j}\left(\alpha_{j} y_{j}-\mathbf{n}_{j}\right) \\
& =(A-Q W) \tilde{x}-G \mathbf{d}+Q \sum_{j} C_{j}^{\prime} Y_{j}^{\prime} Y_{j} \mathbf{n}_{j},
\end{aligned}
$$

where each $Y_{j}$ is a matrix for which $I-\frac{y_{j} y_{j}^{\prime}}{\left\|y_{j}\right\|^{2}}=Y_{j}^{\prime} Y_{j}$. Such matrices always exist because $I-\frac{y_{j} y_{j}^{\prime}}{\left\|y_{j}\right\|^{2}} \geq 0$. Defining $V(\tilde{x}):=\tilde{x}^{\prime} P \tilde{x}, P:=Q^{-1}$ we conclude that

$$
\begin{aligned}
\dot{V} & =\tilde{x}^{\prime}\left(\dot{P}+P A+A^{\prime} P-2 W\right) \tilde{x}-2 \tilde{x}^{\prime} P G \mathbf{d}-2 \tilde{x}^{\prime} \sum_{j} C_{j}^{\prime} Y_{j}^{\prime} Y_{j} \mathbf{n}_{j} \\
& =-\tilde{x}^{\prime}\left(P G G^{\prime} P+W\right) \tilde{x}-2 \tilde{x}^{\prime} P G \mathbf{d}-2 \tilde{x}^{\prime} \sum_{j} C_{j}^{\prime} Y_{j}^{\prime} Y_{j} \mathbf{n}_{j}
\end{aligned}
$$

Here we used (15). By completing the squares, we further conclude that

$$
\begin{aligned}
\dot{V} & =-\frac{1}{2} \tilde{x}^{\prime}\left(P G G^{\prime} P+W\right) \tilde{x}-\frac{1}{2}\left\|G^{\prime} P x+2 \mathbf{d}\right\|^{2}+2\|\mathbf{d}\|^{2}-\sum_{j} \frac{1}{2}\left\|Y_{j} C_{j} \tilde{x}+2 Y_{j} \mathbf{n}_{j}\right\|^{2}+2 \sum_{j} \mathbf{n}_{j}^{\prime} Y_{j}^{\prime} Y_{j} \mathbf{n}_{j} \\
& \leq-\frac{1}{2} \tilde{x}^{\prime}\left(P G G^{\prime} P+W\right) \tilde{x}+2\|\mathbf{d}\|^{2}+2 \sum_{j} \mathbf{n}_{j}^{\prime} Y_{j}^{\prime} Y_{j} \mathbf{n}_{j} \\
& \leq-\frac{1}{2} \tilde{x}^{\prime}\left(P G G^{\prime} P+W\right) \tilde{x}+2\|\mathbf{d}\|^{2}+2 \sum_{j}\left\|\mathbf{n}_{j}\right\|^{2},
\end{aligned}
$$

where we used the fact that the largest eigenvalue of $Y_{j}^{\prime} Y_{j}$ is always smaller than 1 . Since for every finite time $P$ is positive definite, $V$ must be finite on any finite interval and therefore so must be $\tilde{x}$ and $\hat{x}$. Global existence of solution follows. In case $Q$ in uniformly bounded, $P$ is "uniformly" positive definite and so is $P G G^{\prime} P$ (assuming $G G^{\prime} \geq \delta I>0$ ). In this case, it is straightforward to conclude from (21) that the ISS-like bound (19) holds (cf., e.g., [10, Section 5.3]).

Some condition on the observability ${ }^{4}$ of (1)-(2) would be expected to achieve convergence of the estimated state $\hat{x}$ to the process state $x$. In Theorem 2 this condition appear in the form of the requirement that $Q$ remains bounded. In the remaining of this section we investigate conditions under which this happens.

From (7) it is clear that $Q$ remains bounded if $W(t) \geq \epsilon I>0, \forall t \geq 0$ because in this case the term $-Q W Q$ eventually dominates for very large $Q$. However, this case is not very interesting because, e.g., for the single output case $(k=1)$ the matrix $W$ typically has rank equal to ( $\left.\operatorname{rank} C_{1}\right)-1 \leq n-1$ and therefore cannot be nonsingular. The following Lemma provides a significantly weaker condition for the boundedness of $Q$.

Lemma 1. The matrix $Q$ remains bounded along trajectories of the system (1)-(2) and state-estimator (7)-(8), provided that there exist positive constants $T, \epsilon$ such that

$$
\int_{0}^{T} \Phi(t+\tau, t)^{\prime} W(t+\tau) \Phi(t+\tau, t) d \tau \geq \epsilon I>0
$$

$\forall t \geq 0$, where $\Phi(t, \tau)$ denotes the state transition matrix of $\dot{z}=A(u) z$.

\footnotetext{
${ }^{3}$ For simplicity of notation, we will drop the dependence on $u$ of the matrices $A, b, G, C_{j}$ and $d_{j}$.

${ }^{4}$ In the present setup, the correct notion is actually constructability because we are attempting to reconstruct the state from past outputs [1, Section 3.3].
} 
To get some intuition for the meaning of (22) note that for $\int_{0}^{T} \Phi(t+\tau, t)^{\prime} W(t+\tau) \Phi(t+\tau, t) d \tau$ to be singular, there would have to be a vector $x_{0}$ such that

$$
x_{0}^{\prime} \Phi(t+\tau, t)^{\prime} W(t+\tau) \Phi(t+\tau, t) x_{0}=0,
$$

$\forall \tau \in(0, T), t \geq 0$, or equivalently, such that

$$
\beta_{j}(t+\tau) y_{j}(t+\tau)=C_{j}(u(t+\tau)) \Phi(t+\tau, t) x_{0},
$$

$\forall \tau \in(0, T), t \geq 0, j \in\{1, \ldots, k\}$, for appropriate scalars $\beta_{j}(t)$. In essence this means that (22) fails when all the $y_{j}$ evolve as if $u, \mathbf{d}$, and all the $\mathbf{n}_{j}$ were zero. In fact, we can view (22) as a persistence of excitationlike condition that requires $x$ to evolve is some interesting way, other than just following the homogeneous dynamics of (1)-(2), along which scaling information could not be recovered.

It is interesting to note the parallel between the integral in (22) and the constructibility Gramian for linear system [1, Section 3.3]. In fact, if $W$ were replaced by $\sum_{j} C_{j}^{\prime} C_{j}$, the integral in (22) is precisely the constructibility Gramian for the system (1) with linear outputs $C_{j} x+d_{j}+\mathbf{n}_{j}, j \in\{1,2, \ldots, k\}$.

Proof of Lemma 1. To prove this lemma we show that

$$
P(t) \geq \delta I>0, \quad \forall t \geq 0,
$$

for some positive constant $\delta$. To this effect we pick an arbitrary vector $x \in \mathbb{R}^{n}$ and compute $x^{\prime} P(t) x$ using (18):

$$
x^{\prime} P(t) x=z(0)^{\prime} P_{0} z(0)+\int_{0}^{t} z(\tau)^{\prime}\left(P(\tau) G G^{\prime} P(\tau)+W(\tau)\right) z(\tau) d \tau,
$$

where $z(\tau):=\Psi(\tau, t) x, \tau \leq t$ is the solution to

$$
\dot{z}(\tau)=\left(A+G G^{\prime} P\right) z(\tau), \quad z(t)=x, \quad 0 \leq \tau \leq t .
$$

Since $P_{0}>0$, and assuming that $t \geq T$, we conclude that

$$
x^{\prime} P(t) x \geq \alpha(t)^{2}+\beta(t)^{2}, \quad \forall t \geq T,
$$

where

$$
\alpha(t):=\left\|W(\tau)^{\frac{1}{2}} z(\tau)\right\|_{(t-T, t)}, \quad \quad \beta(t):=\left\|G^{\prime} P(\tau) z(\tau)\right\|_{(t-T, t)} .
$$

Here, given a positive semidefinite matrix $M$ we denote by $M^{\frac{1}{2}}$ any matrix such that $\left(M^{\frac{1}{2}}\right)^{\prime} M^{\frac{1}{2}}=M$ and given a signal $x$ we denote by $\|x\|_{(a, b)}$ the $\mathcal{L}_{2}$-norm of $x$ truncated to the interval $(a, b)$, i.e., $\|x\|_{(a, b)}:=$ $\left(\int_{a}^{b}\|x(\tau)\|^{2} d \tau\right)^{\frac{1}{2}}$. We now proceed to compute a lower-bound for $x^{\prime} P(t) x$ by computing a lower-bound for the right-hand-side of $(26)$.

Fix some $t \geq T$. Rewriting (25) as $\dot{z}=A z+G G^{\prime} P z$ and using the variation of constants formula, we conclude that

$$
z(\tau)=\Phi(\tau, t) x+\int_{t}^{\tau} \Phi(\tau, s) G G^{\prime} P(s) z(s) d s, \quad 0 \leq \tau \leq t .
$$

Therefore

$$
\left\|W(\tau)^{\frac{1}{2}} \Phi(\tau, t) x\right\|_{(t-T, t)} \leq \alpha+\left\|\int_{t}^{\tau} W(\tau)^{\frac{1}{2}} \Phi(\tau, s) G G^{\prime} P(s) z(s) d s\right\|_{(t-T, t)}
$$


Moreover, using the Schwartz inequality [13], we conclude that

$$
\begin{aligned}
\left\|\int_{t}^{\tau} W(\tau)^{\frac{1}{2}} \Phi(\tau, s) G G^{\prime} P(s) z(s) d s\right\|_{(t-T, t)}^{2} & =\int_{t-T}^{t}\left\|\int_{t}^{\tau} W(\tau)^{\frac{1}{2}} \Phi(\tau, s) G G^{\prime} P(s) z(s) d s\right\|^{2} d \tau \\
& \leq \int_{t-T}^{t}\left(\int_{\tau}^{t}\left\|W(\tau)^{\frac{1}{2}} \Phi(\tau, s) G\right\|^{2} d s \int_{\tau}^{t}\left\|G^{\prime} P(s) z(s)\right\|^{2} d s\right) d \tau
\end{aligned}
$$

and because $\left\|W(\tau)^{\frac{1}{2}} \Phi(\tau, s) G\right\|$ is uniformly bounded for $s \in[\tau, t]$, we further conclude that

$$
\left\|\int_{t}^{\tau} W(\tau)^{\frac{1}{2}} \Phi(\tau, s) G G^{\prime} P(s) z(s) d s\right\|_{(t-T, t)}^{2} \leq c \int_{t-T}^{t} \int_{\tau}^{t}\left\|G^{\prime} P(s) z(s)\right\|^{2} d s d \tau \leq c T \beta(t)^{2},
$$

for an appropriately defined constant $c$. From (27) and (28), it then follows that

$$
\left\|W(\tau)^{\frac{1}{2}} \Phi(\tau, t) x\right\|_{(t-T, t)} \leq \alpha(t)+\sqrt{c T} \beta(t), \quad \forall t \geq T .
$$

From this, (26), and assuming without loss of generality that $c T \geq 1$, it is straightforward to conclude that

$$
\begin{aligned}
x^{\prime} P(t) x & \geq \alpha^{2}+\beta^{2} \geq \frac{(\alpha+\sqrt{c T} \beta)^{2}}{2 c T} \geq \frac{1}{2 c T}\left\|W(\tau)^{\frac{1}{2}} \Phi(\tau, t) x\right\|_{(t-T, t)}^{2}=\frac{1}{2 c T} \int_{t-T}^{t} x^{\prime} \Phi(\tau, t)^{\prime} W(\tau) \Phi(\tau, t) x d \tau \\
& =\frac{1}{2 c T} x^{\prime} \Phi(t-T, t)^{\prime}\left(\int_{0}^{T} x^{\prime} \Phi(t-T+s, t-T)^{\prime} W(t-T+s) \Phi(t-T+s, t-T) d s\right) \Phi(t-T, t) x,
\end{aligned}
$$

for $t \geq T$. From this and (22), we obtain

$$
x^{\prime} P(t) x \geq \frac{\epsilon}{2 c T}\|\Phi(t-T, t) x\|^{2} \geq \frac{\epsilon}{2 c T\|\Phi(t, t-T)\|^{2}}\|x\|^{2}, \quad \forall t \geq T .
$$

This proves that (24) holds with $\delta$ equal to the smallest of $\frac{\epsilon}{2 c T\|\Phi(t, t-T)\|^{2}}$ and the smallest eigenvalue of $P(t)$ on the closed interval $[0, T]$. The latter is strictly positive since $P(t)$ is positive definite for any finite time $t$.

Combining Theorem 2 and Lemma 1 we obtain the following:

Corollary 1. When Assumption 1 holds and there exist constants $T, \epsilon$ such that (22) holds, the stateestimate $\hat{x}$ converges exponentially fast to the state $x$ in the absence of disturbance input and measurement noise. When the disturbance and noise are bounded but nonzero, $\hat{x}$ may not converge to $x$ but remains bounded away from it.

\section{Output feedback}

We start by considering the set-point control of a perspective linear system (1)-(2) using output-feedback. To this extent, we assume that $A, G$ and all the $C_{j}$ are constant and that

$$
b(u):=B u, \quad \quad d_{j}(u):=D_{j} u, \quad \forall u \in \mathbb{R}^{n_{u}},
$$

for constant matrices $B, D_{j}$. We take a separation approach and assume given a linear state-feedback control law

$$
u=F x+r
$$

that would exponentially stabilize (1) around the equilibriums point $x_{\mathrm{eq}}:=-(A+B F)^{-1} B r$. We thus assume that $A+B F$ is asymptotically stable and therefore that there exist positive definite matrices $R, S$ such that

$$
(A+B F)^{\prime} R+R(A+B F) \leq-S<0 .
$$


Since $x$ cannot be measured we utilize

$$
u=F \hat{x}+r,
$$

instead of (29), where $\hat{x}$ is generated by the state-estimator (7)-(8). In particular,

$$
\dot{\hat{x}}=(A-Q W) \hat{x}+(B-Q w)(F \hat{x}+r) .
$$

We recall that output feedback happens through both $W$ and $w$ that are functions of the outputs $y_{j}$. The following result establishes the validity of the proposed controller.

Theorem 3. When Assumption 1 holds and $Q$ remains uniformly bounded, there exist positive constants $c, \lambda, \gamma_{d}, \gamma_{j}$ such that

$$
\begin{aligned}
& \left\|x(t)-x_{\mathrm{eq}}\right\| \leq e^{-\lambda t}\left\|\left[\begin{array}{l}
x(0)-x_{\mathrm{eq}} \\
\hat{x}(0)-x_{\mathrm{eq}}
\end{array}\right]\right\|+\gamma_{d} \sup _{\tau \in(0, t)}\|\mathbf{d}(\tau)\| \\
& +\sum_{j} \gamma_{j} \sup _{\tau \in(0, t)}\left\|\mathbf{n}_{j}(\tau)\right\|, \quad \forall t>0,
\end{aligned}
$$

along solutions to the closed-loop system (1)-(2), (7)-(8), (31).

Proof of Theorem 3. To analyze the closed-loop system it is convenient to consider a realization of the closeloop system in terms of the state variable $\left[\begin{array}{ll}\tilde{x}^{\prime} & x^{\prime}\end{array}\right]^{\prime}$ with $\tilde{x}:=\hat{x}-x$. Using (20) we conclude that

$$
\left[\begin{array}{c}
\dot{\tilde{x}} \\
\dot{x}
\end{array}\right]=\left[\begin{array}{cc}
A-Q W & 0 \\
B F & A+B F
\end{array}\right]\left[\begin{array}{l}
\tilde{x} \\
x
\end{array}\right]+\left[\begin{array}{l}
0 \\
B
\end{array}\right] r+\left[\begin{array}{c}
-G \mathbf{d}+Q \sum_{j} C_{j}^{\prime}\left(I-\frac{y_{j} y_{j}^{\prime}}{\left\|y_{j}\right\|^{2}}\right) \mathbf{n}_{j} \\
G \mathbf{d}
\end{array}\right] .
$$

The equilibrium can be shifted to the origin by considering the state-variable $z:=\left[\begin{array}{ll}x^{\prime} & x^{\prime}-x_{\mathrm{eq}}^{\prime}\end{array}\right]^{\prime}$ for which

$$
\dot{z}=\left[\begin{array}{cc}
A-Q W & 0 \\
B F & A+B F
\end{array}\right] z+\left[\begin{array}{c}
-G \mathbf{d}+Q \sum_{j} C_{j}^{\prime}\left(I-\frac{y_{j} y_{j}^{\prime}}{\left\|y_{j}\right\|^{2}}\right) \mathbf{n}_{j} \\
G \mathbf{d}
\end{array}\right] .
$$

Considering the Lyapunov function

$$
V(z):=z^{\prime}\left[\begin{array}{cc}
P & 0 \\
0 & \epsilon R
\end{array}\right] z, \quad P:=Q^{-1},
$$

we conclude that $V$ strictly decreases along solutions to the homogeneous part of (33) if

$$
\begin{aligned}
& {\left[\begin{array}{cc}
\dot{P} & 0 \\
0 & 0
\end{array}\right]+\left[\begin{array}{cc}
P & 0 \\
0 & \epsilon R
\end{array}\right]\left[\begin{array}{cc}
A-Q W & 0 \\
B F & A+B F
\end{array}\right]+\left[\begin{array}{cc}
A-Q W & 0 \\
B F & A+B F
\end{array}\right]^{\prime}\left[\begin{array}{cc}
P & 0 \\
0 & \epsilon R
\end{array}\right] } \\
= & {\left[\begin{array}{cc}
\dot{P}+P(A-Q W)+(A-Q W)^{\prime} P & \epsilon F^{\prime} B^{\prime} R \\
\epsilon R B F & \epsilon R(A+B F)+\epsilon(A+B F)^{\prime} R
\end{array}\right]=\left[\begin{array}{cc}
-P G G^{\prime} P-W & \epsilon F^{\prime} B^{\prime} R \\
\epsilon R B F & -\epsilon S
\end{array}\right]<0 . }
\end{aligned}
$$

Here we used (15) and (30). Since $S>0$, a necessary and sufficient condition for this to hold is that

$$
P G G^{\prime} P+W-\epsilon F^{\prime} B^{\prime} R S^{-1} R B F>0,
$$

(cf. [2]) or, in terms of $Q=P^{-1}$,

$$
G G^{\prime}+Q W Q-\epsilon Q F^{\prime} B^{\prime} R S^{-1} R B F Q>0 .
$$

We therefore conclude that if $G G^{\prime} \geq \delta I>0$ and $Q$ remains bounded, it is possible to choose $\epsilon$ sufficiently small so that (34) holds. From (34) it is then straightforward to conclude that (32) holds. 
It is straightforward to generalize the results above to the nonlinear case, provided that the state-feedback controller used is robust (in the input-to-state stability sense) with respect to state perturbations. To this effect, assume given a nonlinear state-feedback control

$$
u=F(x),
$$

that asymptotically stabilizes (1) around an equilibrium $x_{\text {eq }}$ (that we take to be zero without loss of generality). We actually assume that this control is robust with respect to a state perturbation in the sense that (35) makes the system

$$
\dot{x}=A(u) x+b(u)+G(u) \mathbf{d}, \quad u=F(x+\tilde{x})
$$

input-to-state (ISS) with respect to $(\mathbf{d}, \tilde{x})$. Following a separation approach we then use the control

$$
u=F(\hat{x}),
$$

instead of (35), where $\hat{x}$ is generated by the state-estimator (7)-(8). To analyze the system it is convenient to express its dynamics in terms of the state variable $\left[\begin{array}{ll}x^{\prime} & \tilde{x}^{\prime}\end{array}\right]^{\prime}$ with $\tilde{x}:=\hat{x}-x$. Using (20) we conclude that

$$
\begin{array}{ll}
\dot{x}=A(u) x+b(u)+G(u) \mathbf{d}, & u=F(x+\tilde{x}) \\
\dot{\tilde{x}}=(A(u)-Q W) \tilde{x}-G(u) \mathbf{d}+Q \sum_{j} C_{j}^{\prime} Y_{j}^{\prime} Y_{j} \mathbf{n}_{j}, &
\end{array}
$$

To check that this system is ISS with respect to $\left(\mathbf{d}, \mathbf{n}_{1}, \ldots, \mathbf{n}_{k}\right)$ it is sufficient to see that it can be viewed as the cascade of two ISS systems: A system $\Sigma_{1}$ with input $\left(\mathbf{d}, \mathbf{n}_{1}, \ldots, \mathbf{n}_{k}\right)$, state $\tilde{x}$, and dynamics give by (38) that is ISS because of Theorem 2; and a system $\Sigma_{2}$ with input (d, $\left.\tilde{x}\right)$, state $x$, and dynamics (37) that is ISS by assumption. The stability of the cascade of ISS systems has been established, e.g., in [16, 17] (see also [10, Section 5.3]). The following can then be stated:

Theorem 4. When Assumption 1 holds and $Q$ remain uniformly bounded, there exists functions ${ }^{5} \beta \in \mathcal{K} \mathcal{L}$, $\gamma_{d}, \gamma_{1}, \ldots \gamma_{k} \in \mathcal{L}$ such that

$$
\|x(t)\| \leq \beta\left(\left\|\left[x(0)^{\prime} \hat{x}(0)^{\prime}\right]\right\|, t\right)+\sup _{\tau \in(0, t)} \gamma_{d}(\|\mathbf{d}(\tau)\|)
$$

$$
+\sum_{j} \sup _{\tau \in(0, t)} \gamma_{j}\left(\left\|\mathbf{n}_{j}(\tau)\right\|\right), \quad \forall t>0
$$

along solutions to the closed-loop system (1)-(2), (7)-(8), (36).

\section{Rigid body motion estimation using CCD cameras}

In this section we show how one can estimate the position and orientation of a mobile robot using a CCD camera mounted on the robot that observes the apparent motion on the image of stationary points. We do this by reducing the problem to the estimation of the state of a system with projective outputs.

Consider a coordinate frame $\{b\}$ attached to a rigid body that moves with respect to an inertial frame $\{i\}$. We denote ${ }^{6}$ by $\left(p_{i b}, R_{i b}\right) \in \operatorname{SE}(3)$ the configuration of the frame $\{b\}$ with respect to $\{i\}$. Thus, if $q_{1}^{i}$ and $q_{1}^{b}$ denote the coordinates of a point $Q_{1}$ in the frames $\{i\}$ and $\{b\}$, respectively, we have that

$$
q_{1}^{i}=p_{i b}+R_{i b} q_{1}^{b}
$$

\footnotetext{
${ }^{5}$ We denote by $\mathcal{K}$ the set of functions $\alpha:[0, \infty) \rightarrow[0, \infty)$ that are continuous, strictly increasing, and $\alpha(0)=0 ;$ and by $\mathcal{K} \mathcal{L}$ the set of functions $\beta:[0, \infty) \times[0, \infty) \rightarrow[0, \infty)$ for which $\beta(\cdot, t)$ is of class $\mathcal{K}$ for each fixed $t \geq 0$ and $\beta(s, t)$ decreases to 0 as $t \rightarrow \infty$ for each fixed $s \geq 0$.

${ }^{6}$ We denote by $\mathrm{SE}(3)$ the Cartesian product of $\mathbb{R}^{3}$ with the group $\mathrm{SO}(3)$ of $3 \times 3$ rotation matrices; and by se(3) the Cartesian product of $\mathbb{R}^{3}$ with the space so(3) of $3 \times 3$ skew-symmetric matrices (cf.,e.g., [12]).
} 
Moreover, if $q_{j}^{i}$ and $q_{j}^{b}$ denote the coordinates of another point $Q_{j}$ in the frames $\{i\}$ and $\{b\}$, respectively, we conclude that

$$
q_{j}^{b}=R_{i b}^{\prime} q_{j}^{i}-R_{i b}^{\prime} p_{i b}=R_{i b}^{\prime}\left(q_{j}^{i}-q^{i}\right)+q_{1}^{b} .
$$

We denote by $\left(v_{i b}^{b}, \Omega_{i b}^{b}\right) \in \operatorname{se}(3)$ the twist that defines the velocity of frame $\{b\}$ with respect to $\{i\}$, expressed in the frame $\{b\}$, i.e.,

$$
v_{i b}^{b}=R_{i b}^{\prime} \dot{p}_{i b}, \quad \Omega_{i b}^{b}=R_{i b}^{\prime} \dot{R}_{i b} .
$$

From this and (39), we obtain

$$
\dot{q}_{1}^{b}=-\Omega_{i b}^{b} q_{1}^{b}-v_{i b}^{b}+R_{i b}^{\prime} \dot{q}_{1}^{i}, \quad \dot{R}_{i b}=R_{i b} \Omega_{i b}^{b} .
$$

Suppose now that a camera attached to the body frame $\{b\}$ sees $k$ points $Q_{1}, Q_{2}, \ldots, Q_{k}$ rigidly attached to the inertial frame $\{i\}$. Denoting by $y_{j} \in \mathbb{R}^{3}$ the homogeneous image coordinates of the point $Q_{j}$, the dynamics of the system can be described by the following dynamical system with $k$ perspective outputs:

$$
\begin{aligned}
\dot{q}_{1}^{b} & =-\Omega_{i b}^{b} q_{1}^{b}-v_{i b}^{b}, \\
\dot{R}_{i b}^{\prime} & =-\Omega_{i b}^{b} R_{i b}^{\prime}, \\
\alpha_{j} y_{j} & =q_{1}^{b}+R_{i b}^{\prime}\left(q_{j}^{i}-q_{1}^{i}\right), \quad j \in\{1,2, \ldots, k\} .
\end{aligned}
$$

To put this system in the form of (1)-(2) we simply need to define $x$ to be a 12-dimensional vector whose first 3 entries are the entries of $q_{1}^{b}$ and the remaining 9 entries are the columns of $R_{i b}$ stacked on top of each other. Note that once we have estimates $\hat{R}_{i b}$ and $\hat{q}_{1}^{b}$ of $R_{i b}$ and $q_{1}^{b}$, respectively, we can also estimate $p_{i b}$ using

$$
\hat{p}_{i b}=q_{1}^{i}-\hat{R}_{i b} \hat{q}_{1}^{b} .
$$

Figure 5 shows simulation results obtained for a robot following a circular path on the $x-y$ plane, carrying a camera whose optical axis is aligned with the $z$ axis and looks up at four non-coplanar points. The results presented were obtained with and without measurement noise. In the presence of noise, the error in the $z$ coordinate actually does not fully converge to zero. This can be explained because the motion of the robot does not probe the $z$ direction and therefore depth recovery is more sensitive to measurement noise.

\subsection{Singular configurations}

Depending on the configurations of the points $Q_{1}, Q_{2}, \ldots, Q_{k}$, the state of (40)-(42) may not be observable. However, even in this case it may still be possible to recover it by using the fact that $R_{i b}$ is a rotation matrix. To this effect let $M \in \mathbb{R}^{3 \times m}$ be a matrix whose columns are a basis for the vector space generated by the $k-1$ vectors $\left\{q_{j}^{i}-q_{1}^{i}: j=2, \ldots, k\right\}$ and let $\tilde{q}_{j}, j \in\{2, \ldots, k\}$ be such that

$$
q_{j}^{i}-q_{1}^{i}=M \tilde{q}_{j} .
$$

In this case the system (40)-(42) can be re-written as

$$
\begin{aligned}
\dot{q}_{1}^{b} & =-\Omega_{i b}^{b} q_{1}^{b}-v_{i b}^{b}, \\
\dot{N} & =-\Omega_{i b}^{b} N \\
\alpha_{j} y_{j} & =q_{1}^{b}+N \tilde{q}_{j}, \quad j \in\{1,2, \ldots, k\} .
\end{aligned}
$$

where $N:=R_{i b}^{\prime} M \in \mathbb{R}^{3 \times m}$. Note that when $m=\operatorname{rank} M<3$, the system (40)-(42) is not observable because its input-output map is consistent with that of the lower-dimensional model (43)-(45).

To compute an estimate $\hat{R}_{i b}$ of $R_{i b}$ from the estimate $\hat{N}$ of $N$, the following two cases should be considered separately. For simplicity we assume that $M$ was chosen orthonormal, i.e., that $M^{\prime} M=I$. 

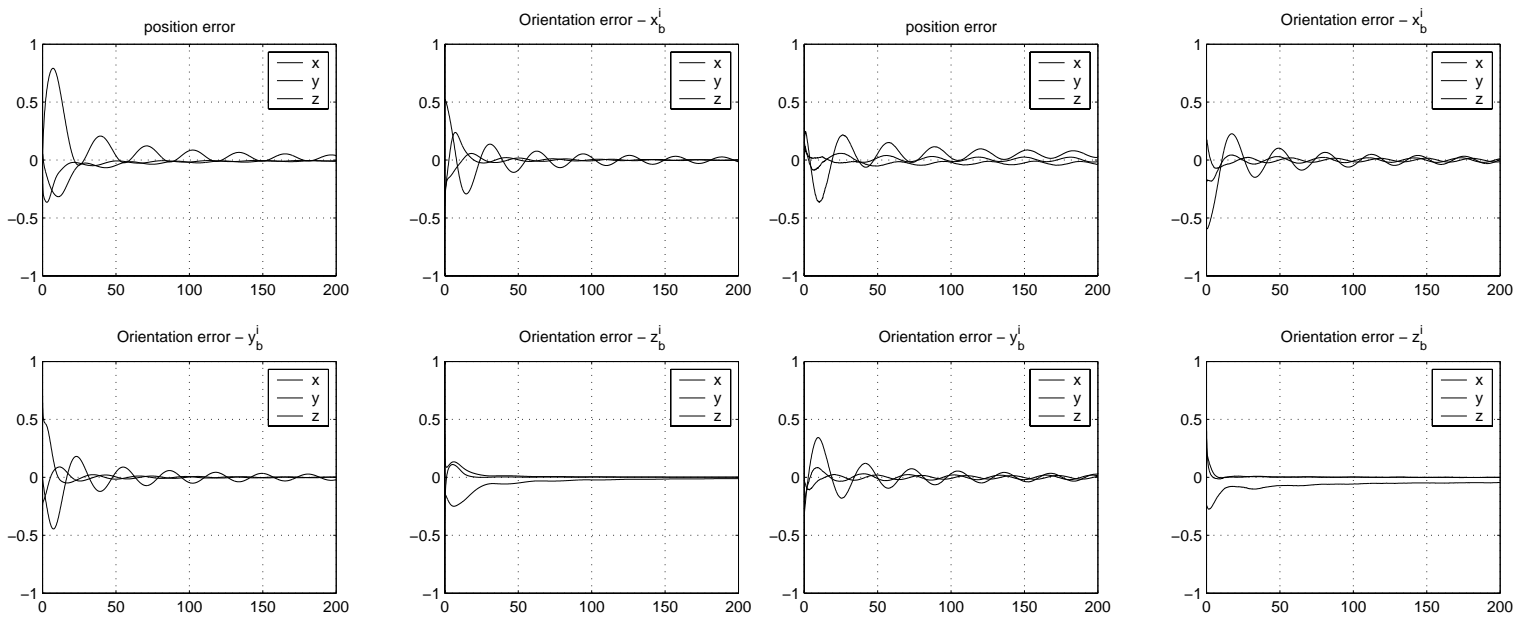

Figure 1: Estimation errors for a simulation in which a robot follows a circular path with a camera looking up at four non-coplanar points. The four plots on the left correspond to no measurement noise, whereas the ones on the right correspond to Gaussian measurement noise with standard deviation equal to roughly $5 \%$ of the measurements. The orientation errors labeled $x_{b}^{i}, y_{b}^{i}$, and $z_{b}^{i}$ correspond to the estimation errors for the first, second, and third columns of $R_{i b}$, respectively.

1. $\operatorname{rank} M=3$, which corresponds to the existence of 4 non-coplanar points. In this case $\hat{R}_{i b}$ can be recovered directly from $\hat{N}$ using

$$
\hat{R}_{i b}^{\prime}=\hat{N} M^{-1}
$$

2. $\operatorname{rank} M<3$, which corresponds to all points being coplanar (rank $M \leq 2$ ) or even collinear ( $\operatorname{rank} M=$ 1). Denoting by $M^{\perp} \in \mathbb{R}^{3 \times(3-m)}$ a matrix whose columns form an orthonormal basis for the orthogonal complement of the image of $M$ (i.e., a full rank matrix such that $M^{\perp^{\prime}} M^{\perp}=I$ and $M^{\prime} M^{\perp}=0$ ), the general solution to $M^{\prime} \hat{R}_{i b}=\hat{N}^{\prime}$, is of the form

$$
\hat{R}_{i b}=M \hat{N}^{\prime}+M^{\perp} \mu^{\prime}
$$

for some vector $\mu \in \mathbb{R}^{3 \times(3-m)}$. This vector needs to be determined from the fact that $\hat{R}_{i b}^{\prime}$ is orthonormal. Since

$$
\begin{aligned}
\hat{R}_{i b}^{\prime} \hat{R}_{i b} & =\left(\hat{N} M^{\prime}+\mu M^{\perp^{\prime}}\right)\left(M \hat{N}^{\prime}+M^{\perp} \mu^{\prime}\right) \\
& =\hat{N} \hat{N}^{\prime}+\mu \mu^{\prime}
\end{aligned}
$$

we conclude that $\mu$ needs to be chosen to make

$$
\hat{N} \hat{N}^{\prime}+\mu \mu^{\prime}
$$

as close to the identity as possible.

For the case, rank $M=2$ (points coplanar but not collinear), it is straightforward to compute the vector $\mu$ that minimizes the Frobenius norm $\|\cdot\|_{F}$ of $\hat{N} \hat{N}^{\prime}+\mu^{\prime} \mu-I$ :

$$
\begin{aligned}
\| \hat{N} \hat{N}^{\prime} & +\mu \mu^{\prime}-I \|_{F}^{2} \\
& :=\operatorname{trace}\left(\hat{N} \hat{N}^{\prime}+\mu \mu^{\prime}-I\right)^{\prime}\left(\hat{N} \hat{N}^{\prime}+\mu \mu^{\prime}-I\right) \\
& =\operatorname{trace} \mu \mu^{\prime} \mu \mu^{\prime}+2 \operatorname{trace}\left(\hat{N} \hat{N}^{\prime}-I\right) \mu \mu^{\prime}+\operatorname{trace}\left(\hat{N} \hat{N}^{\prime}-I\right)^{2} \\
& =\|\mu\|^{4}+2 \mu^{\prime}\left(\hat{N} \hat{N}^{\prime}-I\right) \mu+\operatorname{trace}\left(\hat{N} \hat{N}^{\prime}-I\right)^{2} .
\end{aligned}
$$


Denoting by $\lambda$ the most negative eigenvalue of $\hat{N} \hat{N}^{\prime}-I$ (which must be negative since any vector in the kernel of $\hat{N}^{\prime}$ is an eigenvector corresponding to the eigenvalue -1 ) and by $v$ the corresponding unit-norm eigenvector, the previous expression has a minimum at $\mu^{\prime}=\alpha v$, which is equal to

$$
\alpha^{4}+2 \alpha^{2} \lambda+\operatorname{trace}\left(\hat{N} \hat{N}^{\prime}-I\right)^{2}
$$

where $\alpha$ is a scalar. The minimum is then obtained for $\alpha^{2}=-\lambda$ and therefore $\mu= \pm \sqrt{-\lambda} v$,

$$
\hat{R}_{i b}=M \hat{N}^{\prime} \pm \sqrt{-\lambda} M^{\perp} v^{\prime}
$$

The sign for the square root can be determined from the constrain that the determinant of $\hat{R}_{i b}$ be positive. Note that $\mu$ is unique as long as $\hat{N} \hat{N}^{\prime}-I$ does not have more than one eigenvector associated with the most negative eigenvalue.

\subsection{Unknown inertial coordinates}

Consider now the case in which the inertial coordinates of the points $Q_{1}, Q_{2}, \ldots, Q_{k}$ are not known. In this case, one can still estimate $x$ by using three of the points to define the inertial coordinate system. To this effect, let

$$
S:=R_{i b}^{\prime}\left[\begin{array}{llll}
q_{2}^{i}-q_{1}^{i} & q_{3}^{i}-q_{1}^{i} & \cdots & q_{k}^{i}-q_{1}^{i}
\end{array}\right]
$$

We can re-write $(40)-(42)$ as

$$
\begin{aligned}
\dot{q}_{1}^{b} & =-\Omega_{i b}^{b} q_{1}^{b}-v_{i b}^{b}, \\
\dot{S} & =-\Omega_{i b}^{b} S \\
\alpha_{j} y_{j} & =q_{1}^{b}+S e_{j}, \quad j \in\{1,2, \ldots, k\},
\end{aligned}
$$

where $e_{j}$ denotes the $j$ th column of the $(k-1) \times(k-1)$ identity matrix.

To recover an estimate $\hat{R}_{i b}$ of $R_{i b}$ from the estimate $\hat{S}$ of $S$, we can use the QR decomposition to obtain a rotation matrix $\hat{R}_{i b}$ and an upper triangular matrix $\hat{U}$ such that

$$
\hat{S}=\hat{R}_{i b}^{\prime} \hat{U}
$$

and then defining $q_{1}^{i}:=0$ and $q_{j}^{i}, j \in\{2,3, \ldots, k\}$ equal to the $(j-1)$ th column of $\hat{U}$. This corresponds to the following convention to construct the inertial coordinate system: the origin of $\{i\}$ is the point $Q_{1}$; its first axis is defined by the direction from $Q_{1}$ to $Q_{2}$; its second axis is orthogonal to the first one and lies on the plane defines by $Q_{1}, Q_{2}$, and $Q_{3}$; and its third axis is defined by the cross product of the first two.

\section{Conclusions}

In this paper we considered the problem of estimating the state of a system with perspective outputs. We designed an estimator that is globally convergent under appropriate observability assumptions and can therefore be used to design output-feedback controllers. We applied these results to estimate the position and orientation of a mobile robot using measurements from an attached CCD camera. The estimator proposed requires the robot's linear and angular velocities. Adaptive estimation techniques can probably be used to estimate these parameters. This is the subject of future research. Another topic for future research is to incorporate algebraic constrains on the state in the estimation algorithm. 


\section{References}

[1] P. J. Antsaklis and A. N. Michel. Linear Systems. McGraw-Hill Series in Electrical and Computer Engineering. McGraw-Hill, New York, 1997.

[2] S. Boyd, L. E. Ghaoui, E. Feron, and V. Balakrishnan. Linear Matrix Inequalities in System and Control Theory, volume 15 of SIAM Studies in Applied Mathematics. SIAM, Philadelphia, 1994.

[3] A. Chiuso, P. Favaro, H. Jin, and S. Soatto. Structure from motion causally integrated over time. To appear in IEEE Trans. on Pattern Anal. Mach. Intell., 2002.

[4] W. P. Dayawansa, B. K. Ghosh, C. Martin, and X. Wang. A necessary and sufficient condition for the perspective observability problem. Syst. \& Contr. Lett., 25:159-166, 1995.

[5] B. K. Ghosh, M. Jankovic, and Y. T. Wu. Perspective problems in system theory and its application in machine vision. J. Mathematical Syst. Estimation Contr., 4(1):3-38, 1994.

[6] B. K. Ghosh and E. P. Loucks. A perspective theory for motion and shape estimation in machine vision. SIAM J. Contr. Optimization, 33(5):1530-1559, 1995.

[7] H. Inaba, A. Yoshida, R. Abdursul, and B. K. Ghosh. Observability of perspective dynamical systems. In Proc. of the 39th Conf. on Decision and Contr., volume 5, pages 5157-5162, 2000.

[8] M. Jankovic and Ghosh. Visually guided ranging from observations of points, lines and curves via an identifier based nonlinear observer. Syst. \& Contr. Lett., 25:63-73, 1995.

[9] I. Kaminer, A. M. Pascoal, W. Kang, and O. Yakimenko. Integrated vision/inertial navigation systems design using nonlinear filtering. IEEE Trans. Aerospace and Electronic Syst., 37(1):158-172, Jan. 2001.

[10] H. K. Khalil. Nonlinear Systems. Prentice-Hall, New York, 1996.

[11] L. Matthies, T. Kanade, and R. Szeliski. Kalman filter-based algorithms for estimating depth from image sequences. Int. J. of Computer Vision, 3:209-236, 1989.

[12] R. M. Murray, Z. Li, and S. S. Sastry. A Mathematical Introduction to Robotic Manipulation. CDC Press, Boca Raton, Florida, 1994.

[13] A. W. Naylor and G. R. Sell. Linear Operator Theory in Engineering and Science. Number 40 in Applied Mathematical Sciences. Springer-Verlag, New York, 1982.

[14] H. Rehbinder and B. K. Ghosh. Rigid body state estimation using dynamic vision and inertial sensors. In Proc. of the 40th Conf. on Decision and Contr., volume 3, pages 2398-2403, Dec. 2001.

[15] S. Soatto, R. Frezza, and P. Perona. Motion estimation via dynamic vision. IEEE Trans. on Automat. Contr., 41(3):393-413, Mar. 1996.

[16] E. D. Sontag. Smooth stabilization implies coprime factorization. IEEE Trans. on Automat. Contr., 34 (4):435-443, Apr. 1989.

[17] E. D. Sontag and A. Teel. Changing supply functions in input/state stable systems. IEEE Trans. on Automat. Contr., 40(8):1476-1478, Aug. 1995.

[18] S. Takahashi and B. K. Ghosh. Motion and shape parameters identification with vision and range. In Proc. of the 2001 Amer. Contr. Conf., volume 6, pages 4626-4631, June 2001. 